\title{
Heat Transfer Characteristics of an Inclined Jet on a Roughened Flat Plate
}

\author{
Abdulrahman H. Alenezi, Abdulrahman S. Almutairi, Hamad M. Alhajeri \\ The Public Authority for Applied Education and Training \\ Jamal Abdul Nasser Street, Shuwaikh, Kuwait \\ ah.alenezi@paaet.edu.kw; almutairi76@hotmail.com; hmhajeri@gmail.com
}

\begin{abstract}
Most of the flow ejected from inclined nozzles flows downhill, with less flow travelling in the opposite (uphill) direction. The flow in the uphill direction will become stationary at some radial distance, depending on the jet Reynold's number and the angle of inclination. Designing a rib that maximizes heat transfer rate in the uphill direction was the aim of this research paper. The rib design was based on the idea of enhancing heat transfer in the uphill direction especially for the lower inclined angle $\left(\alpha=45^{\circ}\right)$. The roughened element took the shape dropped tear with square cross-section. The roughness elements were numerically investigated for two angles of jet inclination $\left(\alpha=45^{\circ}\right.$ and $\left.60^{\circ}\right)$ and six different heights $(e / D)$, where $D$ is the hydraulic diameter of the circular jet. The intended nonuniform dropped tear rib shape adapted a design idea of reducing the rib edges in the uphill direction where less flow takes place comparing to the downhill directions. The use of the famous uniform circular rib was for the sake of comparison with the especially designed dropped tear rib based on their effect on the average Nusselt number. The average $\mathrm{Nu}$ were calculated and compared for three cases in this research paper, the no-rib case (the baseline case), the circular rib case (uniform shape) and the dropped tear case (the nonuniform shape) in order to measure the effect of the special design rib on the heta transfer rate. The jet diameter kept constant for all simulations $(D=13.5 \mathrm{~mm})$ where the plane was located at $H / D=6$ below the jet, for all the tests with a jet Reynolds number of 20,000 . Results are presented in the form of average Nusselt number within and beyond the stagnation region.
\end{abstract}

Keywords: Heat transfer, Jet impingement, Inclined jet, Flat plate, Rib.

\section{Introduction}

The nature of the flow in jet impingement applications is difficult to resolve which makes increasing the heat transfer in such applications a complex process. Engineers and scientists have been working for decades to set a range of parameters to study the effect of such parameters on both fluid flow and heat transfer. Adding turbulence promoters is a technique which have been proven to be one of the most effective parameters in increasing the heat transfer in jet impingement applications due to their effect on the flow turbulence.

Andrews, et al., [1] investigated heat transfer for jet impingement for an array of tiny rectangular pin-fins (ribs) which provided 50\% blockage of the crossflow. Up to 15\% heat, transfer enhancement was achieved with rib arrays at a spacing of 3.0. The key factor with such a rib array was its reduction of heat loss due to the crossflow. Xing, et al., [2] studied both experimentally and numerically micro-rib roughened plates with jet impingement under different cross-flow regimes. The micro-rib roughened plate consistently gave a higher heat transfer distribution compared to a smooth flat plate. It was found that the presence of micro-ribs could enhance heat transfer by up to $10 \%$. Caliskan,[3] studied experimentally the effect of using roughened plate on flow heat transfer characteristics. The protrusion elements took the shape of ribs which was organised in to three different configurations. The ribs were also manufactured to either have a solid rib surface (SRS) or perforated rib surface (PRS). The average Nu for impingement plate with PRS configurations shows higher value when compared to SRS impingement plate due the higher flow turbulence resulting from the holes. The maximum enhancement percentage in average $\mathrm{Nu}$ reported by the author is $48 \%$ when using configuration 3 and $\mathrm{H} / \mathrm{D}=2$.

Nuntadusit, et. al., [4] performed an experimental/numerical study to investigate the effect of adapting different types of transverse perforated ribs inside a channel on the heat transfer and flow characteristics. Overall, the maximum heat transfer was achieved using straight perforated rib with hole height of $h=0.2 \mathrm{H}$ due to the jet-like flow behind the protrusion when compared to other rib configurations which sho`wed insignificant heat transfer enhancement. The inclined perforated ribs 
showed significant increase in $\mathrm{Nu}$ when compared to the straight and solid cases, especially at $\theta=30^{\circ}$ which was reported in this study to be the best configuration in enhancing heat transfer.

Wan, et al., [5] studied numerically a square pin-fin configuration on a flat plate with jet impingement. Two configurations were used; inline and staggered. The numerical predictions were in good agreement with the experimental data for both heat transfer and pressure drop. Their results demonstrated the significant benefits to be obtained from using pin-fins on the target plate. Compared to the staggered array, the in-line arrangement achieved 35\% higher heat transfer rate, but with a 3\% reduction of discharge coefficient. In-line pin-fin and staggered pin-fin configurations.

Choi, et. al. [6] in his experimental/numerical study, adapted the effect of both angled ribs and dimples on heat transfer coefficient inside channel using the transient liquid crystal technique. Three surface geometries were adapted in this study, ribbed channel, dimpled channel and rib-dimpled. The author reported that, for all configurations, the higher Re, the better heat transfer.

Donaldson and Snedeker [7,8], studied free jet impingement flow for both normal and oblique conditions with different surface shapes at $\mathrm{H} / \mathrm{D}=39$ for different protrusions shapes. Several combinations of jet flow intensity and jet to target spacings were tested to investigate stagnation pressure for different plate shapes. Figure 2-29. The convex hemisphere shape showed the steepest decline in stagnation pressure with radial distance. In contrast, a concave hemisphere has the least change in stagnation pressure with radial distance.

\section{Numerical Methodology}

Fig. 1 shows the computational domain used in this simulation for both orthogonal and inclined jet impingement which adapted the axisymmetric condition in order to save computational time and cost. All the boundary conditions are shown in the figure. The domain has a diameter of $20 \mathrm{D}$ where $\mathrm{D}$ is the hydraulic diameter. The isothermal wall was kept at a constant temperature of $60{ }^{\circ} \mathrm{C}$ and was impinged by air jet which has an ambient temperature and kept 6D above the heated wall. The simulation parameters are, the jet exit velocity which obtained based on the $\mathrm{Re}=10,000$, the normalized jet-to-target distance (H/D) and the angle of impingement $\alpha$. The chosen values of these parameters were obtained based on the literature where the results of the simulations were validated against the experimental data in [9] for validation. An axisymmetric simulation of jet impingement was carried out using one of the latest versions of the commercial tool ANSYS v18. An initial grid independence study was carried out adapting three different grid sizes in order to find the most suitable grid size that saves computational time and cost. A study was also conducted of the flow and thermal fields employing RNG k- $\varepsilon$, SST k- $\omega$ and RSM turbulence models to investigate their performance. The choice of these models where based on the results reported by other researchers in the literature. The results of the simulations were validated against the experimental data in [9] as mentioned above.

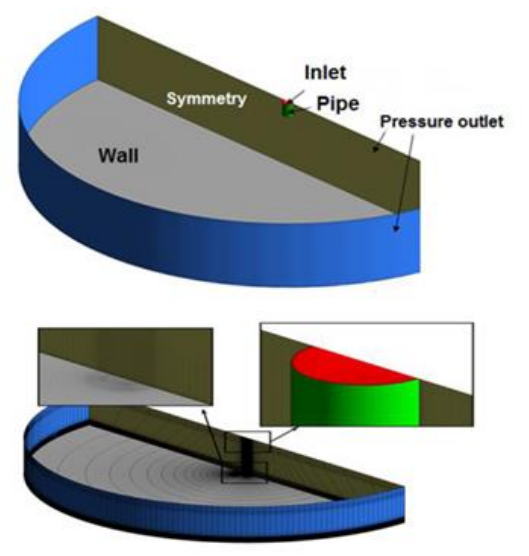

(a)

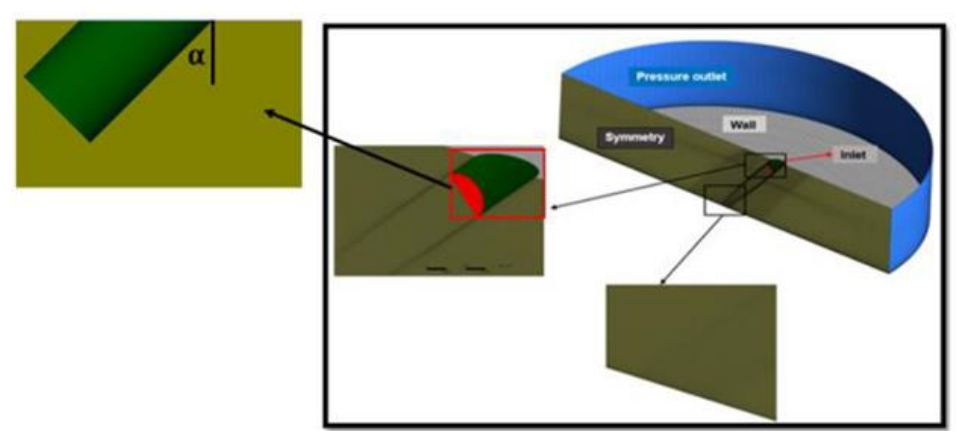

(b)

Fig. 1: Geometry, boundary conditions, and grid for (a) an orthogonal jet impingement, (b) Inclined jet impingement. 


\section{Results}

\subsection{Validation}

Fig shows and compares results obtained from this simulation to results reported from the enamours work done by [9]. The figure demonstrates the local Nu distribution with the radial distance r/D over the heated flat plate. Three grid sizes of 400k, 986k and 1780k were adapted in the simulation keeping $\mathrm{y}+$ equals 0.5 for all grid sizes as recommended by the literature when simulating jet impingement problems.

It is notable in the figure; for $\mathrm{H} / \mathrm{D}=6$ and jet angle $90^{\circ}$, all grid sizes showed good agreement between the numerical and the experimental data and therefore, grid size 2 will be adapted for the rest of the simulations in this paper.

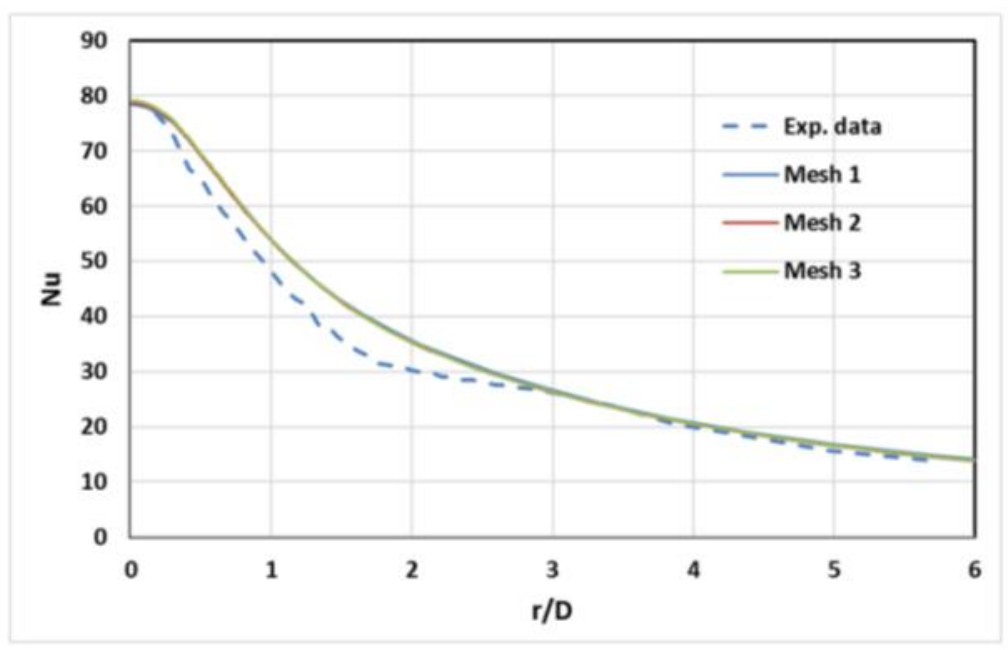

Fig. 2: Nusselt number distribution for three grids, $\mathrm{H} / \mathrm{D}=6, \mathrm{Re}=10,000$.

\subsection{Smooth flat plate (the baseline case).}

As for the effect of Re on heat transfer for inclined jets $\left(\alpha=45^{\circ}\right.$ and $\left.60^{\circ}\right)$, figure 3 presents simulated results for Nu with $\mathrm{H} / \mathrm{D}=6$, respectively, for Reynolds number, $\mathrm{Re}=20,000$ and $\alpha=45^{\circ}$ and $60^{\circ}$ respectively shows that the magnitude of $\mathrm{Nu}$ increases with increase in jet inclination angle. As for the position of the stagnation point, the close jet angle to normal jet, the close the stagnation point to the geometric centre. The secondary peak become more pronounced at higher inclination angle. This phenomenon is also similar for normal jet impingements which are characterized by the fact that the jet is accumulatively delayed thereby building large Reynolds number. Therefore, for a particular H/D setting there will be variations in heat transfer distribution over the impingement plate with change in geometric and jet flow parameters. 


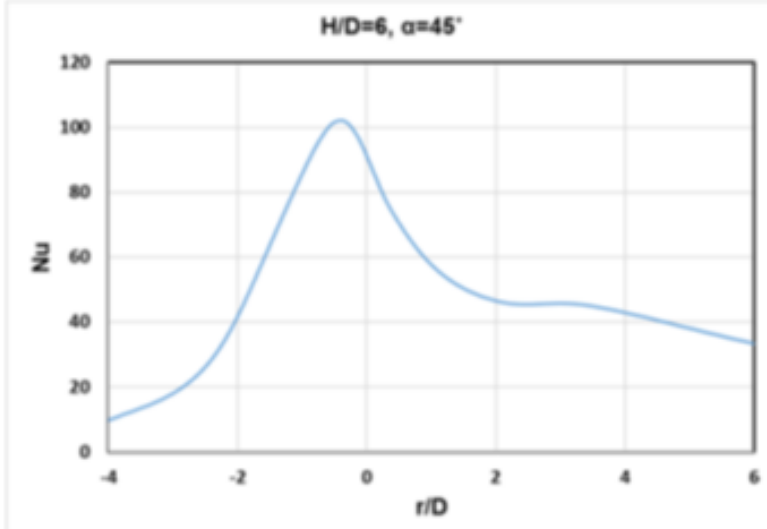

(a)

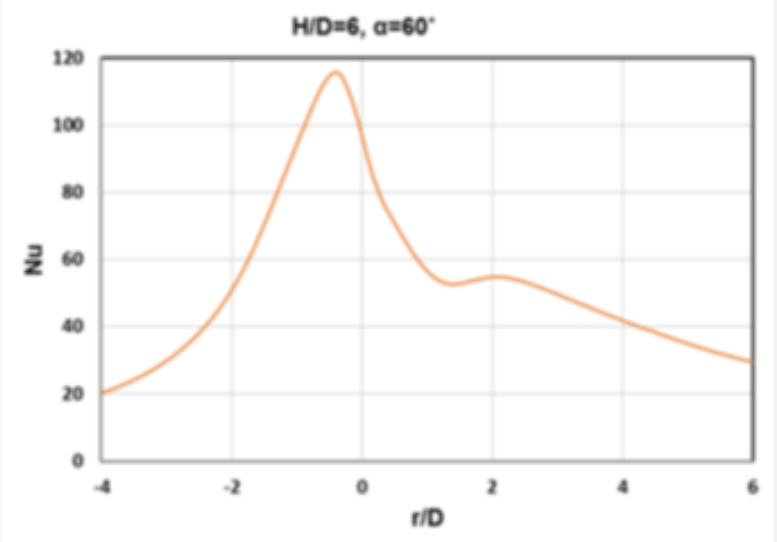

(b)

Fig. 3: Local Nu distribution for $\mathrm{Re}=20,000, \mathrm{H} / \mathrm{D}=6$, (a) $\alpha=45^{\circ}$ and (b) $60^{\circ}$.

\subsection{The circular rib case (the uniform rib)}

The effect of using a roughness element in the shape of a circle with square cross section, and with the same centre as the jet ( see figure 4) on the average $\mathrm{Nu}$ will be investigated. The effectiveness of the roughness element will be tested for different radii $(\mathrm{R} / \mathrm{D})=2$ and six heights (e) between $\mathrm{e}=0.25$ and $1.50 \mathrm{~mm}$ in increments of $0.25 \mathrm{~mm}$. 


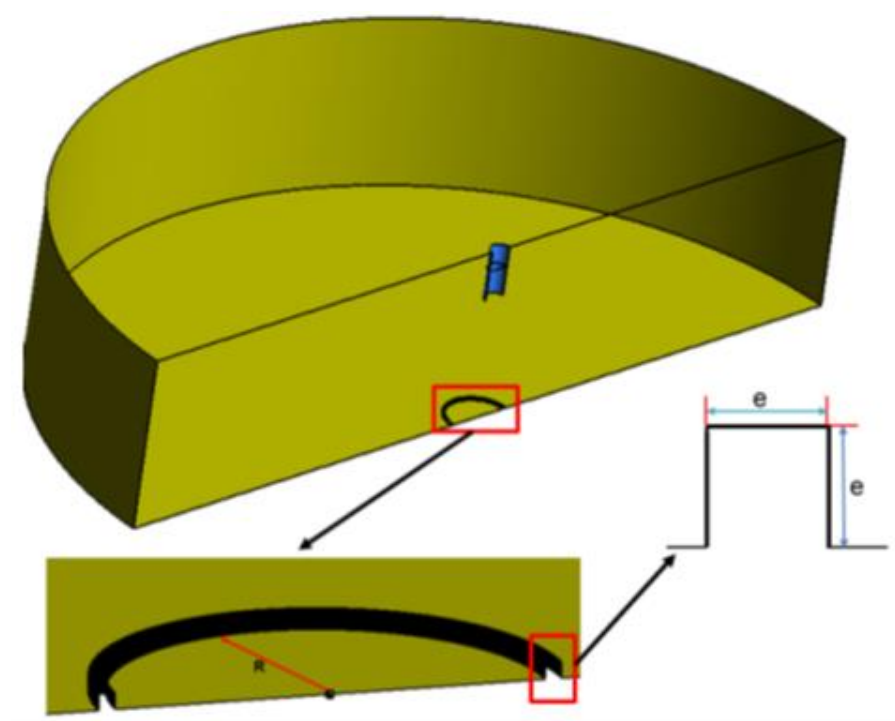

Fig. 4: Geometry of Uniform (circular) rib with square cross-section.

Local $\mathrm{Nu}$ contours for both angles of inclination and rib heights $\mathrm{e}=0.25,0.50$ and $0.75 \mathrm{~mm}$ are shown in figure 5Fig. It is obvious that for $\alpha=45^{\circ}$, all rib heights appear to have a major adverse impact on flow in the uphill direction because the flow hasn't enough momentum to pass over the rib. However, this is not the case for the larger angle of inclination, where there is a greater mass flow rate in the uphill direction. It can also be seen that for $\alpha=60^{\circ}$, the stagnation region is similar for the two rib heights $e=0.25$ and $0.50 \mathrm{~mm}$, but the higher rib of $\mathrm{e}=0.75 \mathrm{~mm}$ shows a different $\mathrm{Nu}$ footprint.
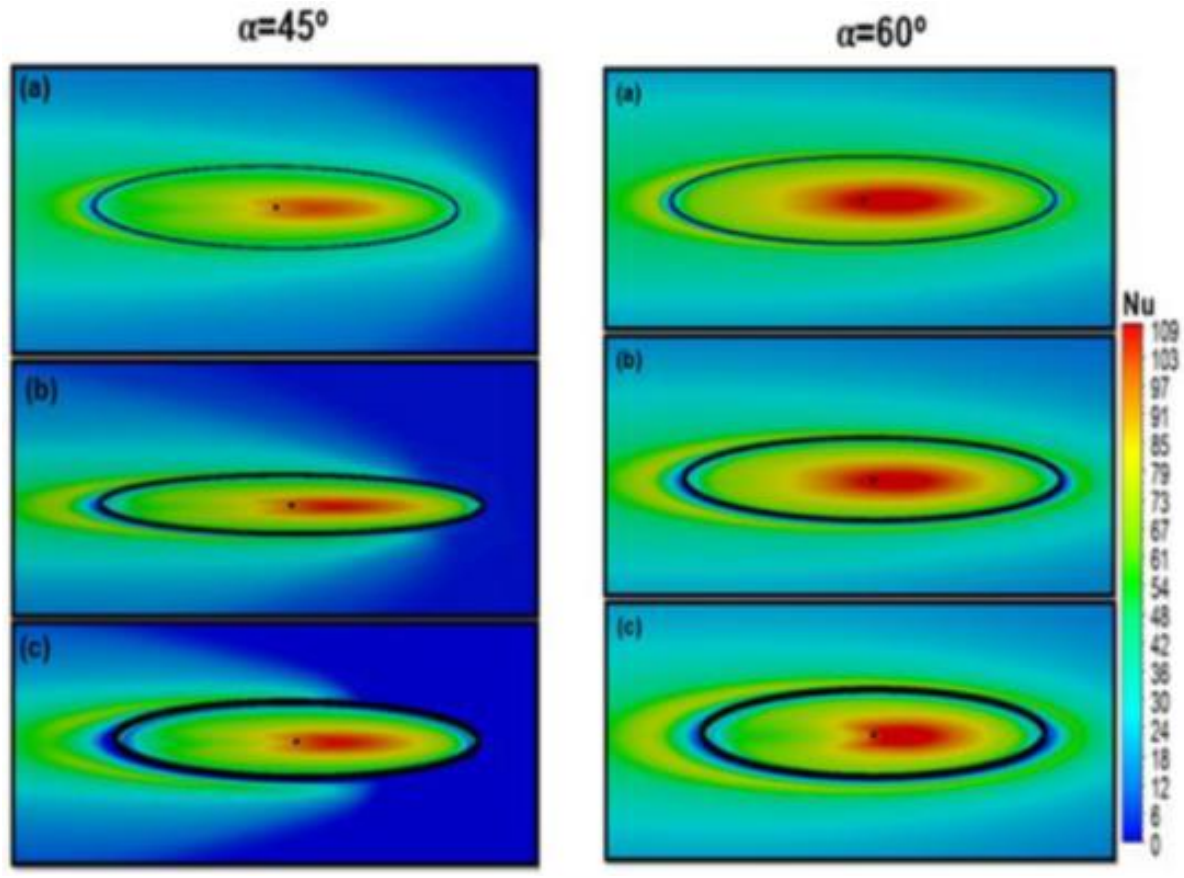

Fig. 5: Local Nu contours for $\mathrm{r} / \mathrm{D}=2$, three heights, (a) $0.25 \mathrm{~mm}$, (b) $0.5 \mathrm{~mm}$ and (c) $0.75 \mathrm{~mm}$ for $\mathrm{Re}=20,000$ and $\mathrm{H} / \mathrm{D}=6$.

The effect on normalized average $\mathrm{Nu}$ of placing the rib at a radial distance $\mathrm{r}=2 \mathrm{D}$, for six rib heights and two jet inclination angles is shown in figure 6. For $\alpha=45^{\circ}$, except for $\mathrm{e}=0.25 \mathrm{~mm}$ which enhanced heat transfer by $3.5 \%$, all ribs showed a lower heat transfer rate than the baseline case. For $\alpha=60^{\circ}$, the rib with height $\mathrm{e}=0.25 \mathrm{~mm}$ also shows an enhancement of the normalized average $\mathrm{Nu}$ of about $3 \%$, the two ribs of heights $0.50 \mathrm{~mm}$ and $0.75 \mathrm{~mm}$ show a similar heat transfer rate 
to the baseline case. Overall, placing the rib at radial distances of $\mathrm{R} / \mathrm{D}=1.5$ and 2 does not seem to be an effective technique to enhance heat transfer for angles of inclination of $45^{\circ}$ and $60^{\circ}$.

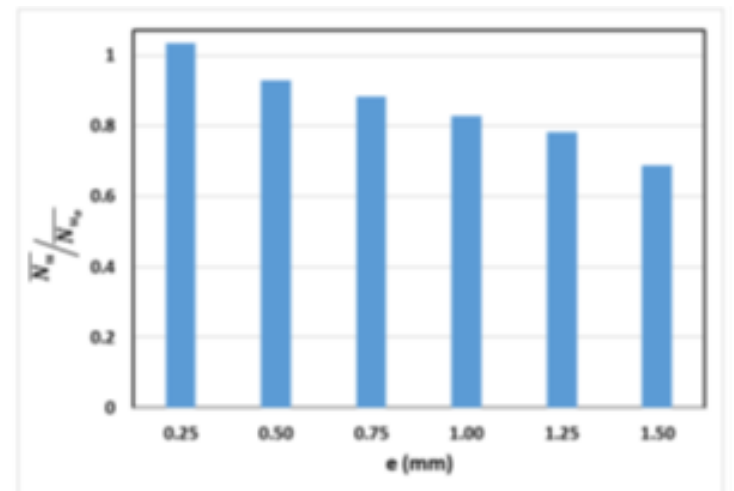

(a)

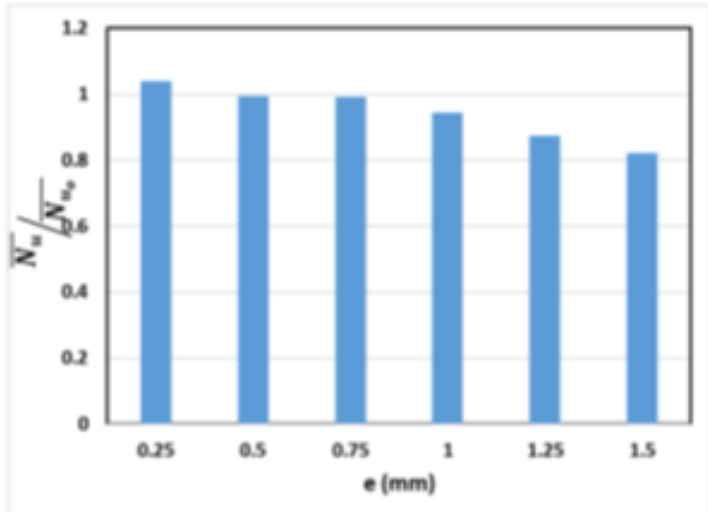

(b)

Fig. 6: Effect of rib height on normalized average $\mathrm{Nu}, \mathrm{Re}=1 \mathrm{D}, \mathrm{Re}, 20,000, \mathrm{H} / \mathrm{D}=6$, (a) $\alpha=45^{\circ}$ and (b) $\alpha=60^{\circ}$.

\subsection{The dropped tear rib case (the non-uniform case)}

Figure 7 shows the non-uniform shapes of the rib used. The rib had six heights as mentioned in the case of a circular rib. The ribs were tested with the geometric centre (G.C.) placed at the dimension shown in the figure. Jet-to-plane distance was $\mathrm{H} / \mathrm{D}=6$, and $\mathrm{Re}=20,000$. The geometric centre is where the flow meets with heated surface when ejected from the nozzle. Two angles of inclination for the jet were used $\alpha=45^{\circ}$ and $60^{\circ}$. The downhill direction is in the direction of $L 2$ for the dropped tear rib. All detailed simulations will be presented for heights of $\mathrm{e}=0.25,0.75$, and $1.50 \mathrm{~mm}$. 

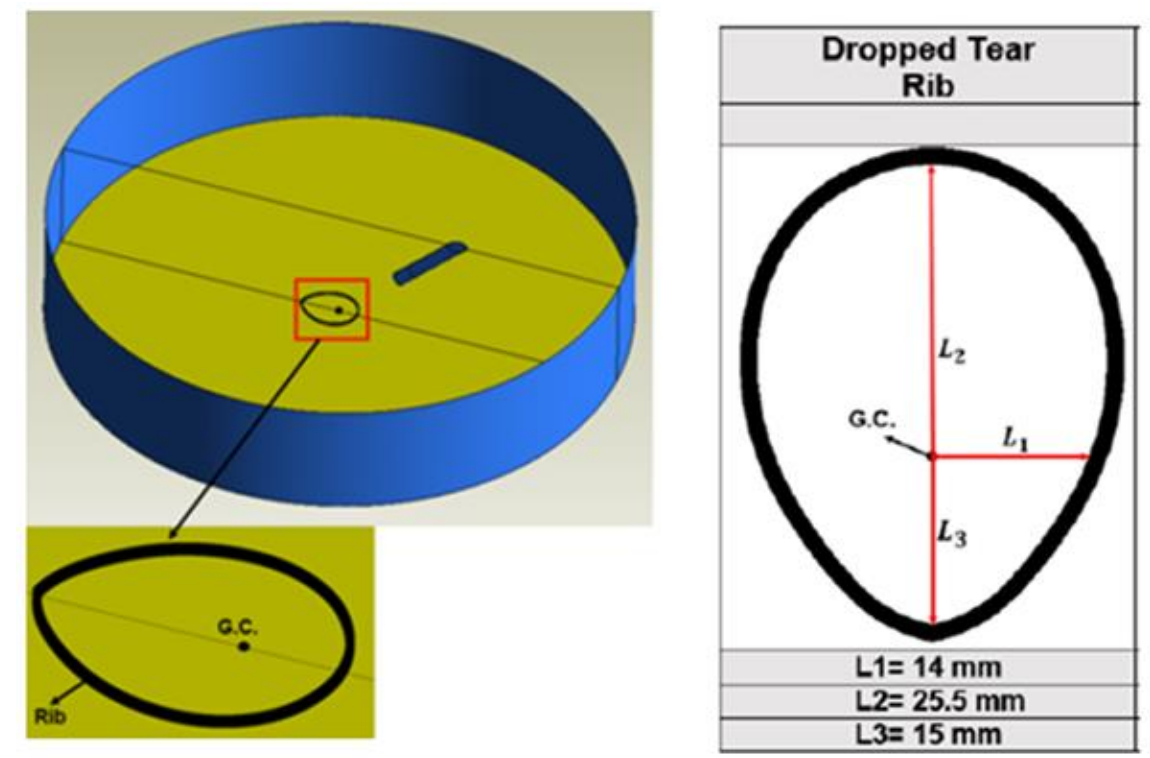

Fig. 7: Geometry details of dropped tear rib.

Fig 8 shows local $\mathrm{Nu}$ contours for dropped tear rib for three rib heights e $=0.25,0.75$ and $1.50 \mathrm{~mm}$ and two angles of inclination of the jet. The sharper end of the rib is located in the uphill direction at a radial distance $L 3=15.0 \mathrm{~mm}(\approx 1.15 \mathrm{D}$, since the nozzle is $13.5 \mathrm{~mm}$ diameter). The figure shows an obvious enhancement in heat transfer especially in the uphill direction. Some enhancement is also noticeable outside the rib due to flow recirculation. In the downhill direction the wake region behind the rib increases the heat transfer. In the uphill direction the wake region after the rib seems reduced and heat transfer is not enhanced so much. For $\alpha=45^{\circ}$, the wake region is minimum when rib height is $\mathrm{e}=0.25 \mathrm{~mm}$ and increases as the rib height increases. However, while the foot-print of the wake shows symmetry, it is non-uniform.

For $\alpha=60^{\circ}$, overall the local $\mathrm{Nu}$ contours in the downhill direction show much the same behaviour as for $\alpha=45^{\circ}$. However, in the uphill direction, the figure shows that for rib heights of $\mathrm{e}=0.75 \mathrm{~mm}$ and $\mathrm{e}=1.50 \mathrm{~mm}$ while there is enhance-ment of local $\mathrm{Nu}$ it is not so great as for the lower angle of inclination.
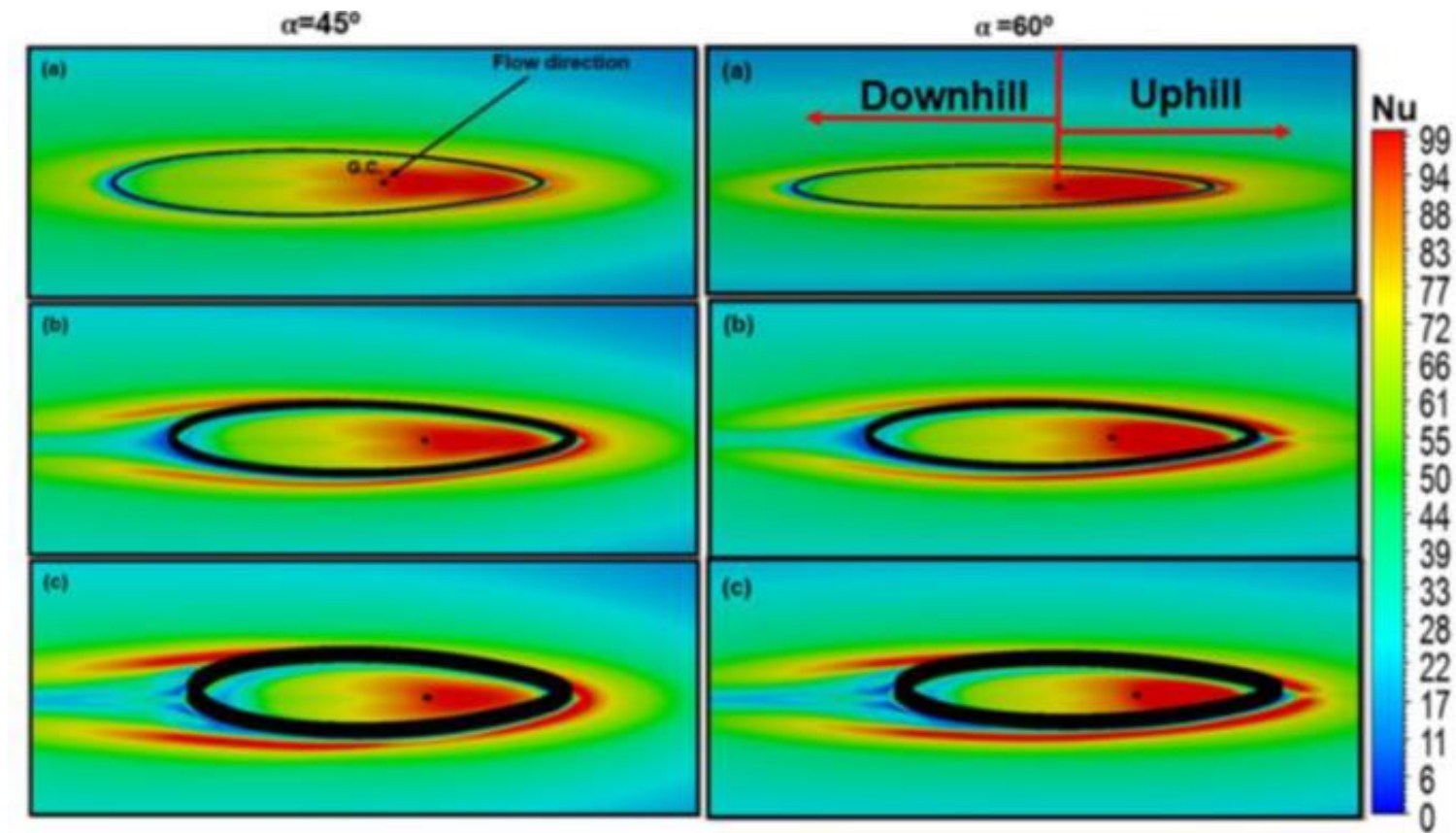

Fig. 8: Local Nu contours for dropped tear rib, $\mathrm{Re}=20,000, \mathrm{H} / \mathrm{D}=6$, (a) $\mathrm{e}=0.25 \mathrm{~mm}$, (b) $\mathrm{e}=0.75 \mathrm{~mm}$ and (c) $\mathrm{e}=1.50 \mathrm{~mm}$. 
The effect of this rib shape on the jet flow was further investigated. Figure 9 shows the turbulence kinetic energy (TKE) contours for three rib heights and two inclined jet angles. The figure shows that the flow turbulence increased in the uphill direction for both angles. This enhancement in flow turbulences is shown by the red region where maximum enhancement can be seen for $\mathrm{e}=0.25 \mathrm{~mm}$ for both angles. In the uphill direction the flow TKE is more pronounced for $\alpha=45^{\circ}$ than for $\alpha=60^{\circ}$. This is due to lower flow velocity in this direction for the lower angle. In the downhill direction, a noticeable enhancement in the flow turbulence is shown behind the roughness element for rib height $\mathrm{e}=0.75 \mathrm{~mm}$, this is more pronounced for $\alpha=45^{\circ}$. For $\mathrm{e}=1.50 \mathrm{~mm}$, the contour footprint shows a continuous wake region downstream the rib, commencing immediately behind the rib. This is due to differences in the flow pressure magnitudes in this region slowing the local flow velocity to almost zero.

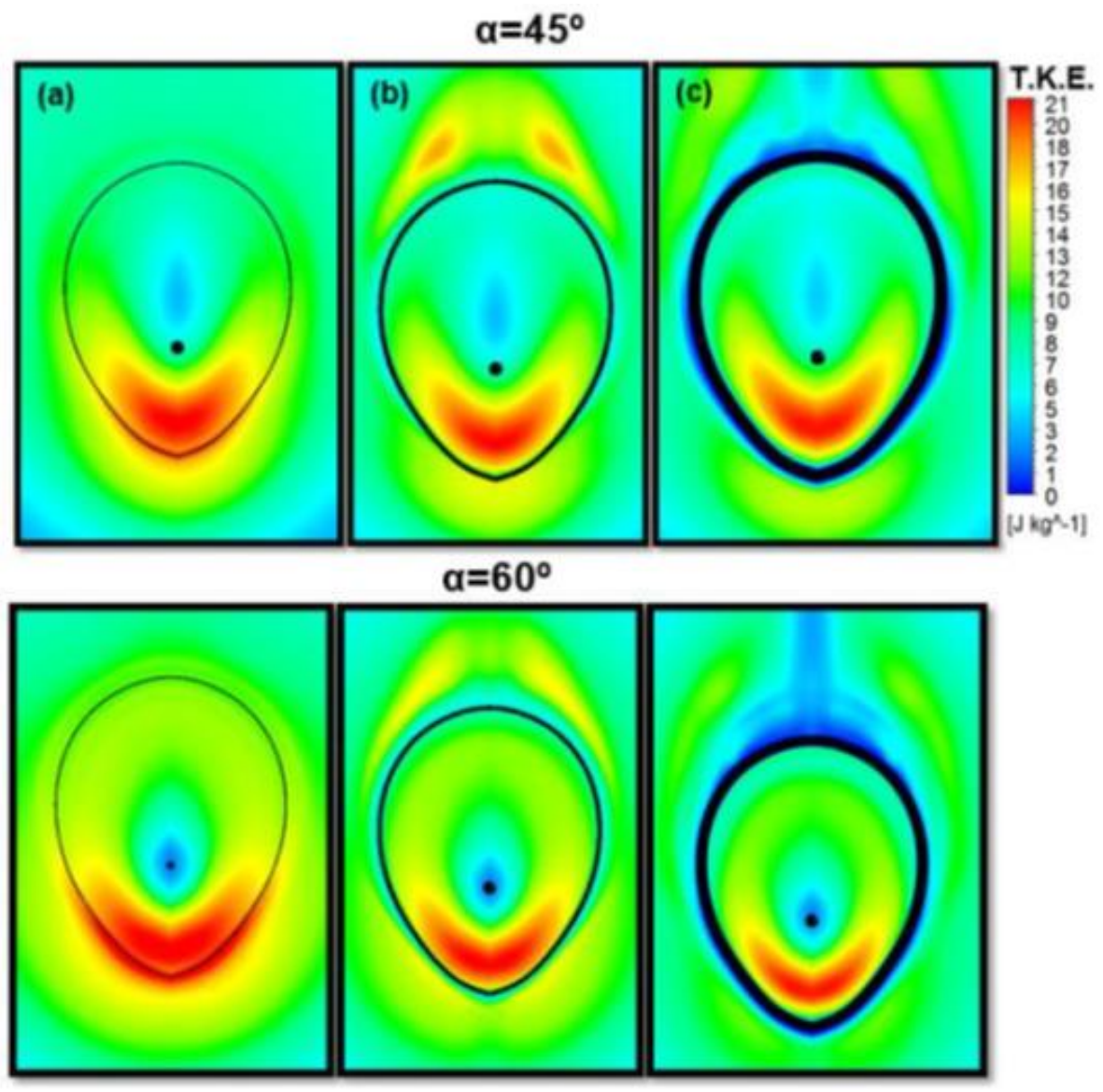

Fig. 9: Plan view of flow turbulence kinetic energy contours for two angles of inclination, $\alpha=45^{\circ}$ and $\alpha=60^{\circ}$. $R e=20,000, H / D=6,(a) e=$ $0.25 \mathrm{~mm}$, (b) $\mathrm{e}=0.75 \mathrm{~mm}$ and (c) $\mathrm{e}=1.50 \mathrm{~mm}$.

The effect of rib height on the normalized average $\mathrm{Nu}$ for $-4 \leq \mathrm{r} / \mathrm{D} \leq 4$ for both inclined jet angles is shown Fig . Changing the plan shape of the rib from a circle to dropped tear gives, overall, a noticeably enhanced heat transfer, as shown in the figure for both angles of inclination of the jet. The lower jet inclination angle shows an overall better average heat transfer enhancement than the higher. The maximum enhancements for $\alpha=45^{\circ}$ and $60^{\circ}$ are $9.5 \%$ and $5.5 \%$, respectively and these occur at rib height $\mathrm{e}=0.25 \mathrm{~mm}$. In addition, unlike the case of a circular rib, with a dropped tear rib shape, all six rib heights show heat transfer enhancement. These are 9.5\%, 8.6\%, 8.4\%, 8.1\%, 7.6\% and $6.3 \%$, respectively for the six rib heights at $\alpha=45^{\circ}$. For $\alpha=60^{\circ}$, the enhancements are between $5.5 \%$ and $2.8 \%$ for rib heights between $0.25 \mathrm{~mm}$ and $1.50 \mathrm{~mm}$. 

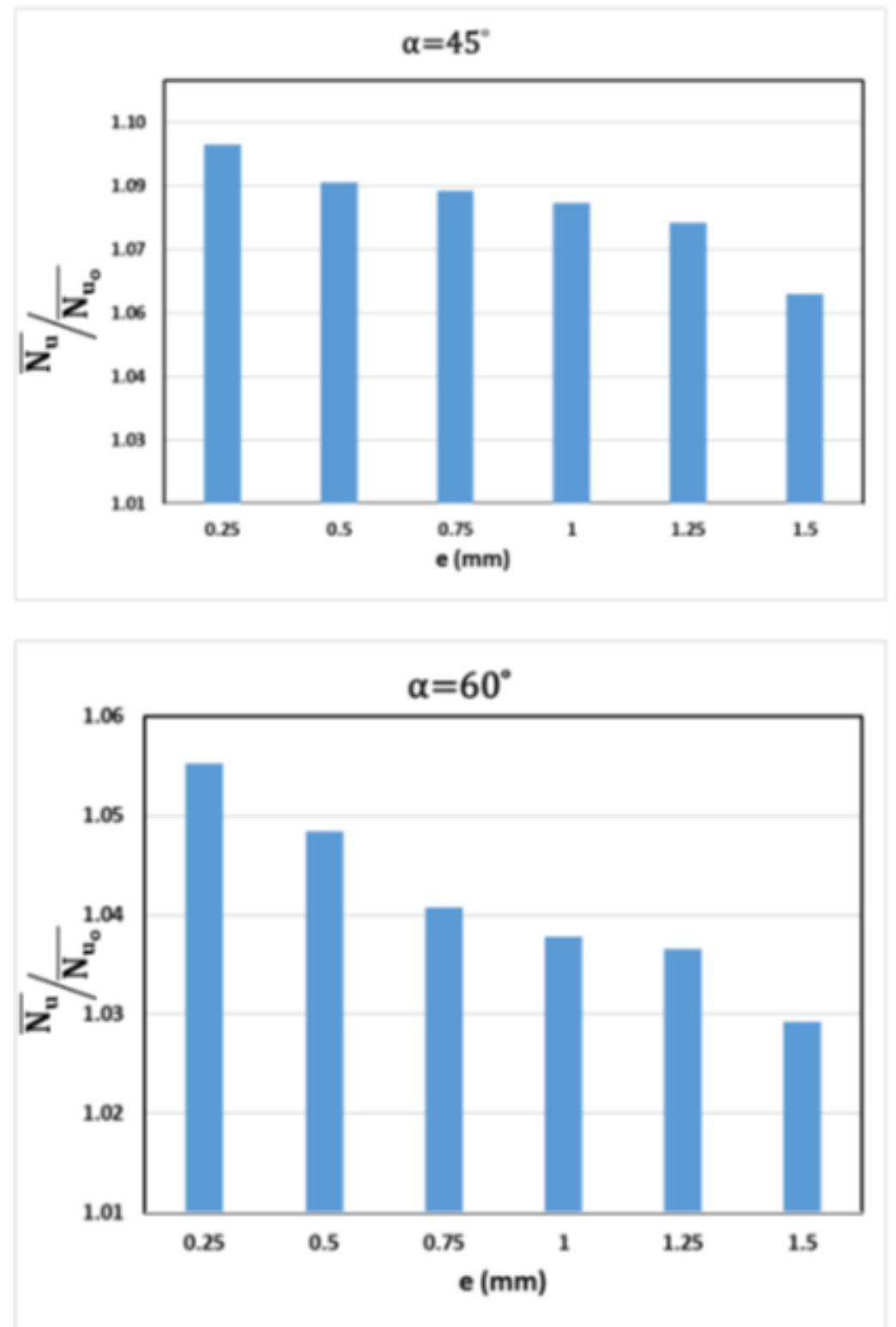

Fig. 10: Effect of rib height on normalized average $\mathrm{Nu}$, dropped tear rib, $\mathrm{Re}=20,000, \mathrm{H} / \mathrm{D}=6$.

\section{Results Comparison}

The heat transfer rate was investigated by determining the normalized average Nusselt number over the surface area between $-4 \leq \mathrm{r} / \mathrm{D} \leq 4$, where most of the heat transfer enhancement occurs. The simulated $\mathrm{Nu}$ was normalized to the simulated average $\mathrm{Nu}$ in the case where no rib was used (the baseline case). The simulation was carried out for two jet inclination angles, $\alpha=45^{\circ}$ and $60^{\circ}, \mathrm{Re}=20,000$, and jet-to-target distance $(\mathrm{H} / \mathrm{D})=6$. Overall results show enhancement in heat transfer rate for both angles but this was more pronounced for $\alpha=45^{\circ}$ than for $60 \mathrm{o}$. For the Circular case, the lower angle of inclination gives a maximum normalized Nusselt number enhancement of 3.5\% where only 3\% enhancement was achieved for the higher angle of inclination. As for the especially designed dropped tear rib shape, the inclination angle $\alpha=45^{\circ}$ showed a maximum enhanced heat transfer of $9.5 \%$ for. However, in the case of $\alpha=60^{\circ}$, the maximum enhancement was only $5.5 \%$. All heat transfer rate enhancements were occurred at rib height $\mathrm{e}=0.25 \mathrm{~mm}$ among all tested rib heights. Table 1 summaries key findings in this research paper. 
Table 1: Optimum normalized $\mathrm{Nu}$ and optimum height for circular and dropped tear rib shapes.

\begin{tabular}{|c|c|c|c|c|}
\hline Parameter & \multicolumn{2}{|c|}{ Circular rib } & \multicolumn{2}{c|}{ Dropped tear rib } \\
\hline$\alpha\left({ }^{\circ}\right)$ & 45 & 60 & 45 & 60 \\
\hline $\mathrm{e}(\mathrm{mm})$ & 0.25 & 0.25 & 0.25 & 0.25 \\
\hline$\overline{N_{u}} / \overline{N_{u_{o}}}$ & $3.5 \%$ & $3 \%$ & $9.5 \%$ & $5.5 \%$ \\
\hline
\end{tabular}

\section{Conclusion}

Most of the flow ejected from inclined nozzles flows downhill, with less flow travelling in the opposite (uphill) direction. The flow in the uphill direction will become stationary at some radial distance, depending on the jet Reynold's number and the angle of inclination. Designing a rib that maximizes heat transfer rate in the uphill direction was the aim of this research paper. The rib design was based on two ideas, (i) placing a roughening element close to the stagnation point, and (ii) have the optimum rib height to maximize flow turbulence. Two rib geometries were tested in this paper, the circular rib and a rib which took dropped tear shape. The non-uniform shape of the rib (the dropped tear rib) was designed to enhance heat transfer in the uphill direction especially for the lower inclined angle where less uphill flow takes place The new rib geometries were designed after an intensive study of the flow velocity contours for inclined jets in order to come up with a design that maximizes the heat transfer between the flow and the heated surface, especially in the uphill direction where the minority of the flow goes.

\section{Acknowledgements}

The authors acknowledge the valuable support and assistance provided by Kuwait Foundation for the Advancement of Sciences (KFAS), in state of Kuwait, through the financial support for this research paper.

\section{References}

[1] G. E. Andrews, R. A. A. Abdul Hussain, M. C. Mkpadi, "Enhanced Impingement Heat Transfer: The Influence of Impingement X/D for Interrupted Rib Obstacles (Rectangular Pin Fins)," ASME Conference Proceedings, pp. 919931, 2004.

[2] Y. Xing, S. Spring, B. Weigand, "Experimental and numerical investigation of impingement heat transfer on a flat and micro-rib roughened plate with different crossflow schemes," International Journal of Thermal Sciences, vol. 50, no. 7, pp. 1293-1307, 2011.

[3] S. Caliskan, "Flow and heat transfer characteristics of transverse perforated ribs under impingement jets," International Journal of Heat and Mass Transfer, vol. 66, pp. 244-260, 2013.

[4] C. Nuntadusit, M. Wae-hayee, A. Bunyajitradulya, S. Eiamsa-ard, "Thermal visualization on surface with transverse perforated ribs," International Communications in Heat and Mass Transfer, vol. 39, no. 5, pp. 634-639, 2012.

[5] C. Wan, Y. Rao, P. Chen, "Numerical predictions of jet impingement heat transfer on square pin-fin roughened plates," Applied Thermal Engineering, vol. 80, pp. 301-309, 2015.

[6] E. Y. Choi, Y. D. Choi, W. S. Lee, J. T. Chung, J. S. Kwak, "Heat transfer augmentation using a rib-dimple compound cooling technique," Applied Thermal Engineering, vol. 51, no. 1-2, pp. 435-441, 2013.

[7] C. D. Donaldson, R. S. Snedeker, "A study of free jet impingement. part 1. mean properties of free and impinging jets," Journal of Fluid Mechanics, vol. 45, no. 2, pp. 281-319, 1971.

[8] C. D. Donaldson, R. S. Snedeker, "A study of free jet impingement. Part 2. Free jet turbulent structure and impingement heat transfer," Journal of Fluid Mechanics, vol. 45, no. 3, pp. 477-512, 1971.

[9] T. S. O’Donovan, B. D. Murray, “Jet impingement heat transfer - Part I: Mean and root-mean-square heat transfer and velocity distributions," International Journal of Heat and Mass Transfer, vol. 50, no. 17-18, pp. 3291-3301, 2007. 